\title{
Communication \\ Stem Carbon Dioxide Efflux of Lignophytes Exceeds That of Cycads and Arborescent Monocots
}

\author{
Thomas E. Marler (D)
}

Citation: Marler, T.E. Stem Carbon Dioxide Efflux of Lignophytes

Exceeds That of Cycads and Arborescent Monocots. Agronomy 2022, 12, 159. https://doi.org/ 10.3390/agronomy12010159

Received: 6 November 2021 Accepted: 7 January 2022

Published: 10 January 2022

Publisher's Note: MDPI stays neutral with regard to jurisdictional claims in published maps and institutional affiliations.

Copyright: (C) 2022 by the author. Licensee MDPI, Basel, Switzerland. This article is an open access article distributed under the terms and conditions of the Creative Commons Attribution (CC BY) license (https:// creativecommons.org/licenses/by/ $4.0 /)$.
Western Pacific Tropical Research Center, University of Guam, Mangilao, GU 96923, USA; marler.uog@gmail.com

\begin{abstract}
Tree stem $\mathrm{CO}_{2}$ efflux (Es) can be substantial and the factors controlling ecosystem-level Es are required to fully understand the carbon cycle and construct models that predict atmospheric $\mathrm{CO}_{2}$ dynamics. The majority of Es studies used woody lignophyte trees as the model species. Applying these lignophyte data to represent all tree forms can be inaccurate. The Es of 318 arborescent species was quantified in a common garden setting and the results were sorted into four stem growth forms: cycads, palms, monocot trees that were not palms, and woody lignophyte trees. The woody trees were comprised of gymnosperm and eudicot species. The Es did not differ among the cycads, palms, and non-palm monocots. Lignophyte trees exhibited Es that was $40 \%$ greater than that of the other stem growth forms. The Es of lignophyte gymnosperm trees was similar to that of lignophyte eudicot trees. This extensive species survey indicates that the Es from lignophyte tree species do not align with the Es from other tree growth forms. Use of Es estimates from the literature can be inaccurate for understanding the carbon cycle in tropical forests, which contain numerous non-lignophyte tree species.
\end{abstract}

Keywords: conservation physiology; secondary cambium; stem respiration

\section{Introduction}

The efflux of carbon dioxide $\left(\mathrm{CO}_{2}\right)$ from tree stem surfaces (Es) has been extensively studied to answer various questions and more fully understand the global carbon cycle [1,2]. As with many aspects of biology research, the Es literature is biased toward one subset of biodiversity. Most case studies of tree Es have focused exclusively on lignophyte species with stems comprised mostly of wood constructed by true bifacial secondary cambium. This expansive literature contains only a few examples in which pachycaulous tree species with stems devoid of bifacial secondary cambium were represented [3-6].

A major contributor to Es is stem tissue respiration. However, numerous interacting factors coalesce to define Es in space and time. For example, $\mathrm{CO}_{2}$ from root respiration can be transported to stems by way of xylem, and this $\mathrm{CO}_{2}$ can exit xylem within stems to increase the Es above that of stem tissue respiration $[7,8]$. This transported $\mathrm{CO}_{2}$ is under the influence of diel variations in sap flow $[9,10]$. The movement of $\mathrm{CO}_{2}$ from the internal tissues to stem surfaces can also be under the control of temporal storage or re-fixation [11]. These and other interacting factors can cause the Es to be heavily influenced by $\mathrm{CO}_{2}$ that was respired from tissues that are distant from the site of efflux [12].

A recent study designed to understand the diel patterns of Es for arborescent cycads, monocots, and lignophytes [6] included only six species of each growth form. Other studies that compared different stem tissue anatomy and its influence in Es were restricted to lignophyte species [13-15]. An extensive survey to compare the Es of trees with disparate stem growth forms has not been conducted to date in a single forest or garden. I hypothesized that Es from an extensive range of tree species would sort into significantly different groups, based on stem design. The objective of this study was to use the large living collection in a common garden setting to compare the Es of four growth forms used to design and construct tree stems. 


\section{Materials and Methods}

This study was conducted at Nong Nooch Tropical Botanical Garden in Sattahip, Thailand. The location and characteristics of this living collection have been described [6]. The dates of measurements were 8-15 July 2019. In this setting and this time of year, the Es of non-lignophyte trees was not influenced by the time of day, but the lignophyte trees exhibited greater Es during midday [6]. Therefore, the measurements for this extensive species survey were restricted to the hours of 900-1500 h on each day of measurement.

A total of 99 cycad species were included (Table A1). There were 96 lignophyte species included (Table A2). The arborescent monocot species were separated into two groups. A total of 17 arborescent monocot taxa that were not palm species were included (Table A3). Finally, there were 106 palm species in the study (Table A4).

The Es was measured, as previously described [4-6]. Vigorous trees with no obvious wounds or decay on the stems were selected. A CIRAS EGM-4 analyzer fitted with a SRC-1 close system chamber (PP Systems, Amesbury, MA, USA) was used to quantify the Es from the stem surfaces. The chamber was secured using modeling clay as the sealant at a stem height of 30-40 cm above the root collar. The EGM-4 recorded the air temperature, and the chamber's increase in $\mathrm{CO}_{2}$ concentration above ambient was quantified after a 2 min period. The change in $\mathrm{CO}_{2}$ concentration was used to calculate the flux by dividing by area and time. Three periods of efflux were recorded at different radial locations for each sampling period for each tree.

The stem surface temperature was measured with an infrared thermometer (Milwaukee Model 2267-20, Milwaukee Tool, Brookfield, WI, USA). The relative humidity was determined with a sling psychrometer every hour during the periods of measurements. The stem diameter at the height of measurements and total stem height were measured for each tree.

Two sampling periods were applied to each species. For taxa with more than one large tree, this included two trees. For taxa with a single large tree, the two samples were from the same tree but separated by at least three days. The data were sorted according to four stem growth forms: cycad species, palm species, arborescent non-palm monocot species, and lignophyte species. The data were subjected to ANOVA using the PROC MIXED model (SAS Institute, Cary, NC, USA) with unequal replications. There were 636 observations in the data set, two per species. The two observations were treated as subsamples in the analysis. The means separation was conducted by Tukey's HSD test.

\section{Results and Discussion}

The cycad trees were represented by 53 Cycadaceae and 46 Zamiaceae species (Table A1). The stem circumference ranged from $51-169 \mathrm{~cm}$ with a mean of $96 \mathrm{~cm}$. The mean stem temperature was $31.8^{\circ} \mathrm{C}$ and the concomitant mean air temperature was $32.6{ }^{\circ} \mathrm{C}$. Individual Es measurements ranged from $0.5-6.2 \mu \mathrm{mol} \cdot \mathrm{m}^{-2} \cdot \mathrm{s}^{-1}$. The lignophyte trees were represented by 34 families (Table A2). The stem circumference ranged from 51-156 cm with a mean of $84 \mathrm{~cm}$. The mean stem temperature was $31.3^{\circ} \mathrm{C}$ and concomitant mean air temperature was $32.0^{\circ} \mathrm{C}$. Individual Es measurements ranged from $0.2-7.6 \mu \mathrm{mol} \cdot \mathrm{m}^{-2} \cdot \mathrm{s}^{-1}$. The monocot trees that were not palm species were represented by five families (Table A3). The stem circumference ranged from $51-175 \mathrm{~cm}$ with a mean of $82 \mathrm{~cm}$. The mean stem temperature was $31.5^{\circ} \mathrm{C}$ and the concomitant mean air temperature was $32.1^{\circ} \mathrm{C}$. The individual Es measurements range from $0.8-4.7 \mu \mathrm{mol} \cdot \mathrm{m}^{-2} \cdot \mathrm{s}^{-1}$. The palm species representing the Arecaceae family exhibited a stem circumference ranging from 48-182 cm with a mean of $71 \mathrm{~cm}$ (Table A4). The mean stem temperature was $31.7^{\circ} \mathrm{C}$ and the concomitant mean air temperature was $32.4{ }^{\circ} \mathrm{C}$. The individual Es measurements ranged from $0.7-7.5 \mu \mathrm{mol} \cdot \mathrm{m}^{-2} \cdot \mathrm{s}^{-1}$. The relative humidity ranged from $56 \%$ to $69 \%$ and did not change substantially among the hours and dates of the study.

The stem $\mathrm{CO}_{2}$ efflux differed among the four stem growth forms $\left(F_{3,314}=10.64\right.$ $p<0.001)$. The means separated into two groups, with the lignophyte species exhibiting greater Es than the other three stem growth forms (Table 1). The lignophyte trees exhibited 
Es that was $40 \%$ greater than the mean of the other growth forms. No differences in the Es occurred among the cycad, palm, and non-palm monocot stem forms.

Table 1. Stem carbon dioxide efflux $\left(\mu \mathrm{mol} \cdot \mathrm{m}^{-2} \cdot \mathrm{s}^{-1}\right)$ of arborescent species as influenced by the stem growth form.

\begin{tabular}{ccc}
\hline Stem Growth Form & n & Efflux \\
\hline Lignophyte $^{1}$ & 96 & $3.421 \pm 0.140 \mathrm{a}^{2}$ \\
Palm & 106 & $2.593 \pm 0.133 \mathrm{~b}$ \\
Cycad & 99 & $2.415 \pm 0.138 \mathrm{~b}$ \\
Monocot (non-palm) & 17 & $2.321 \pm 0.332 \mathrm{~b}$ \\
\hline
\end{tabular}

${ }^{1}$ The lignophyte species were eudicot and gymnosperm trees that produce true wood from secondary bifacial vascular cambium. ${ }^{2}$ Growth form with the same letter not different according to Tukey's HSD test.

Cycads and monocot trees often produce thick primary growth constructed by a primary thickening meristem, and do not possess bifacial secondary cambium to increase stem diameter at distances away from the stem tip [16-22]. For all of these trees, the peripheral tissues are ground tissue with vascular tissues embedded closer to the stem center. One of the factors that influences $\mathrm{CO}_{2}$ efflux from a stem surface is the diffusion and conductance constraints imposed by tissues that are peripheral to tissues that serve as the greatest internal source of $\mathrm{CO}_{2}$, such as sap flow in xylem [23]. The substantial radial distance of xylem tissues and other major sources of $\mathrm{CO}_{2}$ from the stem surface of these pachycaulous trees can account for the greater mean Es for lignophyte trees, which has been shown herein.

Considering the prominence of these pachycaulous trees in tropical forests, the historical exclusion of them from Es studies is unfortunate. Indeed, the $\mathrm{CO}_{2}$ derived from stem efflux can represent up to $40 \%$ of the $\mathrm{CO}_{2}$ contributed to by vegetation [1,24]. This survey, represented by 222 pachycaulous tree species, confirms the earlier findings based on a limited number of species [6], and indicates that attempts to use the Es literature based on the lignophyte species can over-estimate the Es in regions that are represented by these tree species.

Cycads comprise the most threatened contemporary plant group [25]. Conservation physiology has emerged as a critical component of the suite of conservation strategies, because an understanding of the physiological responses of threatened organisms to their escalating biotic and abiotic threats is required for successful species recovery [26,27]. For federally listed endangered cycad species in the United States, such as Cycas micronesica K.D. Hill (see Table A1), understanding the physiology of the taxa is crucial for developing effective federal recovery plans [28]. Clearly, the pursuit of more cycad physiology studies will advance the nascent discipline of conservation physiology.

Future research on the Es of cycad and monocot trees will be required to fully understand the reasons that mean Es is less than the mean Es of lignophyte trees. The design of cycad stems is fairly homogeneous, with vascular cylinders inserted between the persistent living pith and cortex [29]. The design of palm stems is also fairly homogeneous with vascular bundles scattered through the ground tissue [19,22]. However, the design of the non-palm arborescent monocot tree stems is heterogeneous among the families. A closer look at this group of pachycaulous species can yield interesting findings about what endogenous factors mostly control the Es of these non-lignophyte trees.

In conclusion, the many factors that interact to control the magnitude of $\mathrm{CO}_{2}$ efflux from tree stem surfaces are differentially expressed among various tree stem designs. The results herein suggest that the traits of stem peripheral tissues can be among the defining factors that cause the differences in Es among various tree growth forms.

Funding: This research was partly funded by the United States Forest Service, grant numbers 17-DG-11052021-217.

Institutional Review Board Statement: Not applicable. 
Informed Consent Statement: Not applicable.

Data Availability Statement: Data is available in the Appendix.

Acknowledgments: I thank Nong Nooch Tropical Botanical Garden for the logistical support and access to the living collection. I thank Dallas Johnson for the statistical analysis.

Conflicts of Interest: The author declares no conflict of interest. The funders had no role in the design of the study; in the collection, analyses, or interpretation of data; in the writing of the manuscript; or in the decision to publish the results.

\section{Appendix A}

Table A1. List of cycad species included in the carbon dioxide efflux study. Circ = circumference, Air $\mathrm{T}=$ air temperature, and Stem $\mathrm{T}=$ surface temperature of stems.

\begin{tabular}{|c|c|c|c|c|c|c|}
\hline Species & Family & Circ $(\mathrm{cm})$ & Air T & Stem $\mathrm{T}$ & Sample 1 & Sample 2 \\
\hline $\begin{array}{c}\text { Ceratozamia delucana Vázq.Torres, A.Moretti } \\
\text { and Carvajal-Hern. }\end{array}$ & Zamiaceae & 84 & 32 & 32.9 & 2.6222 & 2.3290 \\
\hline Ceratozamia latifolia Miq. & Zamiaceae & 72 & 31 & 32.9 & 2.3227 & 2.0197 \\
\hline Ceratozamia robusta Miq. & Zamiaceae & 112 & 31 & 33.1 & 2.2501 & 1.9566 \\
\hline Cycas angulata R.Br. & Cycadaceae & 107 & 32 & 32.8 & 5.7745 & 5.1166 \\
\hline Cycas apoa K.D.Hill & Cycadaceae & 76 & 33 & 32.5 & 4.8633 & 4.2288 \\
\hline Cycas badensis K.D.Hill & Cycadaceae & 82 & 32 & 31.1 & 1.7512 & 1.5148 \\
\hline Cycas beddomei Dyer & Cycadaceae & 78 & 33 & 31.7 & 1.7819 & 1.5508 \\
\hline Cycas bougainvilleana K.D.Hill & Cycadaceae & 65 & 32 & 31.1 & 4.1522 & 3.6103 \\
\hline Cycas cairnsiana F.Muell. & Cycadaceae & 101 & 31 & 31.4 & 2.0749 & 1.8051 \\
\hline Cycas campestris K.D.Hill & Cycadaceae & 93 & 32 & 30.4 & 2.4442 & 2.1251 \\
\hline Cycas chamaoensis K.D.Hill & Cycadaceae & 101 & 32 & 31.8 & 1.8463 & 1.5969 \\
\hline Cycas changjiangensis N.Liu & Cycadaceae & 81 & 33 & 31.7 & 1.0225 & 2.2722 \\
\hline Cycas clivicola K.D.Hill & Cycadaceae & 87 & 33 & 31.7 & 2.0377 & 1.7805 \\
\hline Cycas couttsiana K.D.Hill & Cycadaceae & 95 & 33 & 32.1 & 0.9089 & 1.1361 \\
\hline Cycas curranii (J.Schust.) K.D.Hill & Cycadaceae & 103 & 32 & 30.1 & 5.1493 & 4.4800 \\
\hline Cycas debaoensis Y.C.Zhong and C.J.Chen & Cycadaceae & 110 & 32 & 30.9 & 1.5203 & 1.3191 \\
\hline Cycas diannanensis Z.T.Guan and G.D.Tao & Cycadaceae & 84 & 33 & 32.1 & 5.6678 & 4.9231 \\
\hline Cycas edentata de Laub. & Cycadaceae & 68 & 32 & 29.8 & 1.9212 & 1.6663 \\
\hline Cycas elongata (Leandri) D.Y.Wang & Cycadaceae & 103 & 32 & 31.9 & 2.1460 & 1.7042 \\
\hline Cycas falcata K.D.Hill & Cycadaceae & 89 & 33 & 31.8 & 2.8403 & 1.4517 \\
\hline Cycas furfuracea W.Fitzg. & Cycadaceae & 108 & 34 & 34.5 & 2.5564 & 2.2217 \\
\hline Cycas glauca Miq. & Cycadaceae & 92 & 33 & 32.2 & 4.0045 & 3.5346 \\
\hline $\begin{array}{c}\text { Cycas hainanensis C.J.Chen ex C.Y.Cheng, } \\
\text { W.C.Cheng and L.K.Fu }\end{array}$ & Cycadaceae & 91 & 33 & 31.4 & 2.5496 & 2.2217 \\
\hline Cycas hongheensis S.Y.Yang and S.L.Yang & Cycadaceae & 82 & 32 & 30.8 & 2.2847 & 2.1165 \\
\hline Cycas inermis Lour. & Cycadaceae & 109 & 33 & 32.1 & 6.1934 & 5.4154 \\
\hline Cycas javana (Miq.) de Laub. & Cycadaceae & 112 & 33 & 32.7 & 2.7945 & 2.4300 \\
\hline Cycas macrocarpa Griff. & Cycadaceae & 75 & 31 & 31.2 & 4.1164 & 3.6103 \\
\hline Cycas media R.Br. & Cycadaceae & 82 & 33 & 31.9 & 2.1964 & 1.8996 \\
\hline Cycas megacarpa K.D.Hill & Cycadaceae & 62 & 33 & 31.8 & 2.1114 & 1.8380 \\
\hline Cycas micronesica K.D.Hill & Cycadaceae & 62 & 33 & 32.5 & 1.3764 & 1.1992 \\
\hline Cycas nathorstii J.Schust. & Cycadaceae & 102 & 32 & 31.9 & 1.7446 & 1.5148 \\
\hline Cycas nongnoochiae K.D.Hill & Cycadaceae & 88 & 33 & 31.9 & 2.9513 & 2.5689 \\
\hline Cycas ophiolitica K.D.Hill & Cycadaceae & 115 & 33 & 32.1 & 1.1846 & 1.0225 \\
\hline Cycas pachypoda K.D.Hill & Cycadaceae & 98 & 32 & 30.8 & 4.4112 & 3.8482 \\
\hline Cycas papuana F.Muell. & Cycadaceae & 86 & 31 & 30.7 & 5.3312 & 4.6517 \\
\hline Cycas pectinata Buch.-Ham. & Cycadaceae & 93 & 32 & 33.4 & 2.0547 & 1.7805 \\
\hline Cycas petrae A.Lindstr. and K.D.Hill & Cycadaceae & 69 & 33 & 32.1 & 1.7965 & 2.3353 \\
\hline Cycas platyphylla K.D.Hill & Cycadaceae & 116 & 33 & 31.7 & 0.6817 & 1.5905 \\
\hline $\begin{array}{c}\text { Cycas pranburiensis Yang, Tang, Hill and } \\
\text { Vatcharakorn }\end{array}$ & Cycadaceae & 78 & 33 & 32.5 & 1.5115 & 1.3210 \\
\hline Cycas revoluta Thunb. & Cycadaceae & 85 & 32 & 32.8 & 2.2223 & 1.9314 \\
\hline
\end{tabular}


Table A1. Cont.

\begin{tabular}{|c|c|c|c|c|c|c|}
\hline Species & Family & Circ (cm) & Air T & Stem $\mathrm{T}$ & Sample 1 & Sample 2 \\
\hline Cycas riuminiana Porte ex Regel & Cycadaceae & 65 & 33 & 33.1 & 3.7784 & 3.2821 \\
\hline Cycas rumphii Miq. & Cycadaceae & 84 & 30 & 32.1 & 4.0561 & 3.5346 \\
\hline Cycas seemannii A.Br. & Cycadaceae & 63 & 32 & 31.1 & 3.0296 & 2.1523 \\
\hline Cycas semota K.D.Hill & Cycadaceae & 84 & 33 & 30.2 & 3.4865 & 3.0296 \\
\hline Cycas shanyaensis G.A.Fu & Cycadaceae & 68 & 32 & 32.1 & 2.4690 & 3.0296 \\
\hline Cycas siamensis Miq. & Cycadaceae & 79 & 32 & 31.9 & 3.4431 & 2.9867 \\
\hline Cycas silvestris K.D.Hill & Cycadaceae & 69 & 33 & 32.2 & 1.6679 & 1.4517 \\
\hline Cycas sphaerica Roxb. & Cycadaceae & 92 & 32 & 30.4 & 3.4155 & 2.9867 \\
\hline Cycas taitungensis Shen, Hill, Tsou and Chen & Cycadaceae & 83 & 32 & 31.8 & 4.4478 & 3.8880 \\
\hline Cycas tansachana K.D.Hill and S.L.Yang & Cycadaceae & 113 & 33 & 31.8 & 1.1864 & 1.0099 \\
\hline Cycas thouarsii R.Br. & Cycadaceae & 82 & 31 & 30.9 & 1.1968 & 1.0225 \\
\hline Cycas tropophylla K.D.Hill and P.K.Lôc & Cycadaceae & 94 & 33 & 32.1 & 2.0312 & 1.7673 \\
\hline Cycas tuckeri K.D.Hill & Cycadaceae & 95 & 32 & 30.8 & 1.7157 & 1.4933 \\
\hline Cycas wadei Merr. & Cycadaceae & 83 & 32 & 31.3 & 2.1113 & 1.8380 \\
\hline Cycas yorkiana K.D.Hill & Cycadaceae & 94 & 33 & 32.1 & 2.1564 & 1.8746 \\
\hline $\begin{array}{c}\text { Cycas zeylanica (J.Schust.) A.Lindstr. and } \\
\text { K.D.Hill }\end{array}$ & Cycadaceae & 78 & 33 & 30.8 & 2.3312 & 2.0103 \\
\hline $\begin{array}{c}\text { Dioon argenteum Gregory, Chemnick, } \\
\text { Salas-Morales and Vovides }\end{array}$ & Zamiaceae & 93 & 33 & 31.1 & 4.9455 & 4.4308 \\
\hline Dioon caputoi De Luca, Sabato and Vázq.Torres & Zamiaceae & 91 & 32 & 34.8 & 2.4857 & 2.1523 \\
\hline Dioon edule Lindl. & Zamiaceae & 155 & 32 & 33.1 & 3.7795 & 3.2821 \\
\hline Dioon mejiae Standl. and L.O. Williams & Zamiaceae & 98 & 33 & 31.6 & 1.9594 & 1.7042 \\
\hline Dioon merolae De Luca, Sabato and Vázq.Torres & Zamiaceae & 110 & 32 & 32.7 & 4.9132 & 4.2920 \\
\hline Dioon spinulosum Dyer ex Eichl. & Zamiaceae & 91 & 33 & 32.2 & 2.2561 & 1.9566 \\
\hline Encephalartos aemulans Vorster & Zamiaceae & 138 & 33 & 31.9 & 4.4466 & 3.9976 \\
\hline Encephalartos altensteinii Lehm. & Zamiaceae & 116 & 34 & 32.2 & 2.2504 & 2.4679 \\
\hline Encephalartos arenarius R.A.Dyer & Zamiaceae & 97 & 33 & 32.2 & 1.4495 & 1.5905 \\
\hline Encephalartos bubalinus Melville & Zamiaceae & 125 & 33 & 31.7 & 1.0569 & 1.1614 \\
\hline $\begin{array}{c}\text { Encephalartos chimanimaniensis R.A.Dyer and } \\
\text { I.Verd. }\end{array}$ & Zamiaceae & 121 & 33 & 31.8 & 1.5948 & 1.7420 \\
\hline Encephalartos concinnus R.A.Dyer and Verdoorn & Zamiaceae & 114 & 33 & 32.1 & 4.9765 & 5.4465 \\
\hline $\begin{array}{c}\text { Encephalartos dyerianus Lavranos and } \\
\text { D.L.Goode }\end{array}$ & Zamiaceae & 124 & 34 & 32.9 & 3.5004 & 3.8501 \\
\hline Encephalartos equatorialis P.J.H.Hurter & Zamiaceae & 127 & 34 & 32.9 & 1.7764 & 1.9314 \\
\hline Encephalartos eugene-maraisii Verd. & Zamiaceae & 116 & 33 & 32.1 & 2.2465 & 2.4679 \\
\hline Encephalartos inopinus R.A.Dyer & Zamiaceae & 121 & 34 & 32.2 & 2.3611 & 2.5878 \\
\hline Encephalartos lebomboensis I.Verd. & Zamiaceae & 97 & 33 & 32.3 & 1.6564 & 1.8146 \\
\hline Encephalartos mackenziei L.E.Newton & Zamiaceae & 111 & 33 & 32.2 & 0.8645 & 0.9089 \\
\hline Encephalartos macrostrobilus S.Jones and Wyants & Zamiaceae & 125 & 34 & 32.8 & 1.7946 & 1.9566 \\
\hline Encephalartos manikensis (Gilliland) Gilliland & Zamiaceae & 133 & 33 & 31.7 & 1.5946 & 1.7420 \\
\hline Encephalartos msinganus Vorster & Zamiaceae & 108 & 33 & 32.1 & 2.9764 & 3.2663 \\
\hline Encephalartos munchii R.A.Dyer and I.Verd. & Zamiaceae & 134 & 33 & 32.2 & 2.3331 & 2.5562 \\
\hline Encephalartos natalensis R.A.Dyer and I.Verd. & Zamiaceae & 109 & 34 & 32.9 & 1.1915 & 1.3065 \\
\hline $\begin{array}{c}\text { Encephalartos paucidentatus Stapf and Burtt } \\
\text { Davy }\end{array}$ & Zamiaceae & 120 & 33 & 32.5 & 1.7764 & 1.9314 \\
\hline Encephalartos princeps R.A.Dyer & Zamiaceae & 113 & 33 & 32.8 & 2.0645 & 1.8947 \\
\hline Encephalartos pterogonus R.A.Dyer and I.Verd. & Zamiaceae & 119 & 33 & 33.1 & 2.4465 & 2.6986 \\
\hline $\begin{array}{c}\text { Encephalartos sclavoi De Luca, D.W.Stev. and } \\
\text { A.Moretti }\end{array}$ & Zamiaceae & 101 & 33 & 32.1 & 1.5959 & 1.7420 \\
\hline Encephalartos senticosus Vorster & Zamiaceae & 107 & 32 & 30.9 & 0.8465 & 0.8710 \\
\hline Encephalartos septentrionalis Schweinf. & Zamiaceae & 119 & 34 & 32.9 & 1.6694 & 3.7239 \\
\hline Encephalartos tegulaneus Melville & Zamiaceae & 124 & 33 & 32.1 & 3.6915 & 4.0647 \\
\hline Encephalartos transvenosus Stapf and Burtt Davy & Zamiaceae & 128 & 33 & 31.2 & 4.5121 & 4.9357 \\
\hline Encephalartos whitelockii P.J.H.Hurter & Zamiaceae & 163 & 32 & 31.1 & 2.7154 & 2.9865 \\
\hline Lepidozamia hopei (W.Hill) Regel & Zamiaceae & 64 & 32 & 29.1 & 1.4896 & 1.2623 \\
\hline Lepodozamia peroffskyana Regel & Zamiaceae & 92 & 32 & 33.8 & 1.6765 & 1.8304 \\
\hline
\end{tabular}


Table A1. Cont.

\begin{tabular}{|c|c|c|c|c|c|c|}
\hline Species & Family & Circ (cm) & Air T & Stem $\mathbf{T}$ & Sample 1 & Sample 2 \\
\hline Macrozamia moorei F.Muell. & Zamiaceae & 169 & 32 & 32.7 & 1.8686 & 1.6663 \\
\hline Microcycas calocoma (Miq.) A.DC. & Zamiaceae & 91 & 32 & 32.1 & 2.0644 & 1.8304 \\
\hline $\begin{array}{c}\text { Zamia elegantissima Schutzman, Vovides and } \\
\text { R.S.Adams }\end{array}$ & Zamiaceae & 51 & 33 & 30.9 & 1.3312 & 1.4517 \\
\hline Zamia furfuracea L.f. & Zamiaceae & 91 & 33 & 32.1 & 1.7154 & 1.8746 \\
\hline Zamia gentryi Dodson & Zamiaceae & 63 & 33 & 30.5 & 2.0105 & 2.1999 \\
\hline $\begin{array}{c}\text { Zamia imperialis A.S.Taylor, J.L.Haynes and } \\
\text { Holzman }\end{array}$ & Zamiaceae & 59 & 32 & 31.1 & 1.3866 & 1.5148 \\
\hline Zamia lindenii Regel ex André & Zamiaceae & 78 & 30 & 29.9 & 2.0166 & 2.1998 \\
\hline Zamia obliqua A.Braun & Zamiaceae & 51 & 32 & 30.2 & 2.7626 & 2.9965 \\
\hline Zamia skinneri Warsc. & Zamiaceae & 58 & 32 & 30.2 & 2.3465 & 2.5765 \\
\hline
\end{tabular}

Table A2. List of the lignophyte species included in the carbon dioxide efflux study. Circ = circumference, Air T = air temperature, and Stem $\mathrm{T}=$ surface temperature of stems.

\begin{tabular}{|c|c|c|c|c|c|c|}
\hline Species & Family & Circ (cm) & Air T & Stem $\mathbf{T}$ & Sample 1 & Sample 2 \\
\hline Acacia auriculiformis A.Cunn. Ex Benth. & Fabaceae & 92 & 32 & 29.3 & 3.5566 & 3.7944 \\
\hline Adansonia digitata $\mathrm{L}$. & Malvaceae & 71 & 31 & 31.4 & 3.9465 & 3.5346 \\
\hline Adansonia madagascariensis Baill. & Malvaceae & 69 & 33 & 32.3 & 2.0566 & 1.8304 \\
\hline Afrocarpus gracilior (Pilg.) C.N. Page & Podocarpaceae & 72 & 32 & 31.3 & 6.4465 & 6.3748 \\
\hline Agathis dammara (Lamb.) Rich. & Araucariaceae & 85 & 33 & 32.2 & 2.3465 & 2.5698 \\
\hline Agathis moorei (Lind.) Mast. & Araucariaceae & 52 & 33 & 30.9 & 1.3255 & 1.8304 \\
\hline Agathis robusta (C.Moore ex F.Muell.) Bailey & Araucariaceae & 55 & 32 & 30.9 & 1.7198 & 1.8935 \\
\hline Albizia saman (Jacq.) Merr. & Fabaceae & 156 & 32 & 30.6 & 1.2765 & 1.3886 \\
\hline Amherstia nobilis Wall. & Fabaceae & 68 & 32 & 31.4 & 2.1894 & 1.9566 \\
\hline Annona squamosa L. & Annonaceae & 62 & 31 & 30.8 & 1.6768 & 1.8746 \\
\hline Araucaria bidwillii Hook. & Araucariaceae & 91 & 32 & 31.2 & 5.0032 & 4.4813 \\
\hline Araucaria columnaris J.R.Forst. Hook & Araucariaceae & 69 & 30 & 30.2 & 6.2264 & 5.6237 \\
\hline Araucaria cunninghamii Mudie & Araucariaceae & 58 & 31 & 29.4 & 2.3631 & 2.0829 \\
\hline Araucaria heterophylla (Salisb.) Franco & Araucariaceae & 96 & 32 & 33.2 & 2.8403 & 2.7771 \\
\hline $\begin{array}{c}\text { Araucaria luxurians (Brongn. and Grisb.) de } \\
\text { Laub. }\end{array}$ & Araucariaceae & 64 & 31 & 30.1 & 6.0021 & 5.4281 \\
\hline Araucaria montana Brong. and Gris & Araucariaceae & 54 & 32 & 31.8 & 2.9034 & 2.6643 \\
\hline Araucaria nemorosa de Laub. & Araucariaceae & 93 & 33 & 31.8 & 2.0829 & 2.0197 \\
\hline Artocarpus altilis (Parkinson) Fosberg & Moraceae & 88 & 33 & 32.1 & 3.6625 & 3.9986 \\
\hline Artocarpus heterophyllus Lam. & Moraceae & 74 & 31 & 30.8 & 2.4689 & 2.7140 \\
\hline Averrhoa bilimbi L. & Oxalidaceae & 72 & 33 & 32.3 & 4.2165 & 4.6647 \\
\hline Averrhoa carambola $\mathrm{L}$ & Oxalidaceae & 69 & 31 & 30.1 & 4.4465 & 3.9865 \\
\hline Bougainvillea sp. Comm. Ex Juss. & Nyctaginaceae & 51 & 33 & 32.1 & 1.9955 & 2.1133 \\
\hline $\begin{array}{c}\text { Brachychiton acerifolius (A.Cunn ex G.Don) } \\
\text { F.Muell. }\end{array}$ & Malvaceae & 53 & 31 & 31.7 & 2.2566 & 2.0134 \\
\hline $\begin{array}{c}\text { Brachychiton rupestris (T.Mitch. Ex Lindl.) } \\
\text { K.Schum. }\end{array}$ & Malvaceae & 54 & 30 & 30.3 & 4.4656 & 4.0269 \\
\hline Bursera simaruba (L.) Sarg. & Burseraceae & 51 & 32 & 30.8 & 0.8119 & 0.9026 \\
\hline Callistemon viminalis (Sol. Ex Gaertn.) G.Don & Myrtaceae & 77 & 30 & 30.7 & 6.0022 & 5.4154 \\
\hline Callistris baileyi C.T. White & Cupressaceae & 59 & 31 & 30.8 & 2.6644 & 2.3984 \\
\hline Calophyllum sil Lauterb. & Clusiaceae & 103 & 30 & 30.9 & 3.3186 & 3.0107 \\
\hline Cananga odorata (Lam.) Hook.f. and Thomson & Annonaceae & 121 & 32 & 31.1 & 4.4212 & 4.8766 \\
\hline Casuarina equisitifolia $\mathrm{L}$. & Casuarinaceae & 102 & 33 & 33.4 & 3.5564 & 3.8965 \\
\hline Cavanillesia hylogeiton Ulbr. & Malvaceae & 111 & 33 & 32.8 & 2.8845 & 2.5562 \\
\hline Cecropia obtusifolia Bertol. & Urticaceae & 88 & 31 & 30.3 & 5.1114 & 4.6012 \\
\hline Cecropia peltata L. & Urticaceae & 113 & 32 & 31.6 & 3.6266 & 3.2265 \\
\hline Ceiba pentandra (L.) Gaertn. & Malvaceae & 123 & 33 & 31.9 & 4.3489 & 3.9196 \\
\hline Clusia rosea Jacq. & Clusiaceae & 61 & 33 & 31.3 & 3.1153 & 3.5642 \\
\hline Delonix decaryi (R.Vig.) Capuron & Fabaceae & 82 & 32 & 31.7 & 1.3349 & 1.1992 \\
\hline
\end{tabular}


Table A2. Cont.

\begin{tabular}{|c|c|c|c|c|c|c|}
\hline Species & Family & Circ (cm) & Air T & Stem $\mathbf{T}$ & Sample 1 & Sample 2 \\
\hline Delonix regia (Hook.) Raf. & Fabaceae & 72 & 32 & 31.2 & 3.6644 & 3.2947 \\
\hline Dimocarpus longan Lour. & Sapindaceae & 126 & 33 & 32.3 & 2.8844 & 2.5649 \\
\hline Diospyros discolor Willd. & Ebenaceae & 129 & 33 & 31.7 & 0.5116 & 0.4418 \\
\hline Diospyros nigra (J.F.Gmel.) Perrier & Ebenaceae & 99 & 30 & 28.9 & 4.1445 & 4.5444 \\
\hline Elaeocarpus hygrophilus Kurz & Elaeocarpaceae & 53 & 31 & 30.8 & 3.9787 & 3.5699 \\
\hline Euphorbia kamponii Rauh and Petignat & Euphorbiaceae & 53 & 33 & 33.2 & 1.1899 & 1.3065 \\
\hline Euphorbia laeta Aiton & Euphorbiaceae & 81 & 34 & 33.5 & 4.8011 & 4.2920 \\
\hline Fernandoa madagascariensis (Baker) A.H.Gentry & Bignoniaceae & 51 & 31 & 30.3 & 2.0645 & 1.8304 \\
\hline Ficus benjamina $\mathrm{L}$. & Moraceae & 151 & 30 & 29.8 & 3.2233 & 2.9034 \\
\hline Ficus elastica Roxb. ex Hornem. & Moraceae & 94 & 33 & 32.2 & 3.6151 & 3.2663 \\
\hline Ficus lyrata Warb. & Moraceae & 104 & 32 & 31.4 & 6.1184 & 5.8793 \\
\hline Ficus natalensis Hochst. & Moraceae & 56 & 31 & 29.8 & 5.6112 & 5.0083 \\
\hline $\begin{array}{c}\text { Garcinia cymosa (K.Schum.) I.M.Turner and } \\
\text { P.F.Stevens }\end{array}$ & Clusiaceae & 64 & 32 & 31.3 & 3.2311 & 2.9760 \\
\hline Guaiacum officinale $\mathrm{L}$. & Zygophyllaceae & 74 & 32 & 30.5 & 4.3365 & 3.8956 \\
\hline Inga edulis Mart. & Fabaceae & 64 & 33 & 32.2 & 1.0848 & 0.9650 \\
\hline Kopsia arborea Blume & Apocynaceae & 78 & 31 & 30.8 & 1.1323 & 0.9976 \\
\hline Lagerstroemia indica $\mathrm{L}$. & Lythraceae & 66 & 33 & 31.9 & 5.7112 & 5.1125 \\
\hline Lagerstroemia speciosa (L.) Pers. & Lythraceae & 78 & 33 & 32.1 & 6.3054 & 5.6616 \\
\hline Leucaena leucocephala (Lam.) de Wit & Fabaceae & 51 & 32 & 30.8 & 2.0202 & 2.2091 \\
\hline Litsea elliptica Blume & Lauraceae & 62 & 32 & 31.3 & 2.1132 & 1.8935 \\
\hline Magnolia $\times$ alba (D.C.) Figlar & Magnoliaceae & 145 & 32 & 31.8 & 1.6650 & 1.5148 \\
\hline Mallotus barbatus Müll.Arg. & Euphorbiaceae & 82 & 30 & 30.3 & 5.7765 & 5.1347 \\
\hline Mangifera foetida Lour. & Anacardiaceae & 57 & 33 & 33.4 & 7.4986 & 6.9429 \\
\hline Mangifera indica $\mathrm{L}$. & Anacardiaceae & 61 & 33 & 33.5 & 4.0065 & 3.5847 \\
\hline Melaleuca bracteata F. Muell. & Myrtaceae & 96 & 31 & 30.2 & 1.0065 & 1.4466 \\
\hline Morinda citrifolia L. & Rubiaceae & 61 & 30 & 30.7 & 1.3389 & 1.4678 \\
\hline Moringa hildebrandtii Eng. & Moringaceae & 82 & 32 & 31.2 & 4.1132 & 3.6608 \\
\hline Moringa oleifera Lam. & Moringaceae & 75 & 33 & 32.1 & 0.2211 & 0.1894 \\
\hline Muntingia calabura L. & Muntingiaceae & 66 & 32 & 31.1 & 1.5644 & 1.3886 \\
\hline Nephelium lappaceum L. & Sapindaceae & 128 & 33 & 32.3 & 1.8686 & 1.6663 \\
\hline Nerium oleander L. & Apocynaceae & 52 & 32 & 30.8 & 1.2234 & 1.1321 \\
\hline Pachira aquatica Aub. & Malvaceae & 105 & 33 & 32.1 & 2.0044 & 1.7988 \\
\hline Pachira insignis (Sw.) Savigny & Malvaceae & 95 & 30 & 30.6 & 5.2123 & 5.8068 \\
\hline Persea americana Mill. & Lauraceae & 126 & 33 & 32.5 & 1.5590 & 1.3232 \\
\hline Phyllanthus acidus (L.) Skeels & Phyllanthaceae & 86 & 32 & 31.1 & 1.4391 & 1.5565 \\
\hline Pithecellobium dulce (Roxb.) Benth. & Fabaceae & 96 & 32 & 31.8 & 1.6265 & 1.4517 \\
\hline Plumeria rubra L. & Apocynaceae & 73 & 33 & 31.6 & 5.3795 & 5.9764 \\
\hline Podocarpus neriifolius D.Don & Podocarpaceae & 85 & 30 & 29.8 & 4.3035 & 4.7965 \\
\hline Polyalthia longifolia (Sonn.) Thwaites & Annonaceae & 93 & 33 & 32.1 & 3.1138 & 2.7105 \\
\hline Pouteria campechiana (Kunth.) Baehni & Sapotaceae & 138 & 32 & 30.7 & 1.1133 & 2.0197 \\
\hline Pseudobombax septenatum (Jacq.) Dugand & Malvaceae & 66 & 31 & 30.7 & 0.9922 & 0.8836 \\
\hline Psidium guajava L. & Myrtaceae & 52 & 30 & 30.8 & 4.9597 & 5.5416 \\
\hline Robinia hispida L. & Fabaceae & 66 & 33 & 31.8 & 2.4465 & 2.2247 \\
\hline Sandoricum koetjape (Burm.f.) Merr. & Meliaceae & 141 & 33 & 32.1 & 1.9322 & 1.7042 \\
\hline Saraca asoca (Roxb.) Willd. & Fabaceae & 72 & 31 & 30.3 & 4.1645 & 4.5679 \\
\hline Saraca declinata Miq. & Fabaceae & 75 & 32 & 30.1 & 3.0111 & 3.3452 \\
\hline Saraca thaipingensis Prain & Fabaceae & 89 & 31 & 30.7 & 1.0946 & 1.1992 \\
\hline Schizolobium parahyba (Vll.) S.F.Blake & Fabaceae & 91 & 32 & 31.1 & 0.9955 & 0.8836 \\
\hline $\begin{array}{c}\text { Senegalia polyacantha (Willd.) Siegler and } \\
\text { Ebinger }\end{array}$ & Fabaceae & 81 & 33 & 31.8 & 3.7989 & 4.2265 \\
\hline Sterculia foetida $\mathrm{L}$ & Malvaceae & 141 & 33 & 32.4 & 3.4844 & 3.8877 \\
\hline Syzygium cumini (L.) Skeels & Myrtaceae & 151 & 32 & 30.2 & 4.6075 & 3.9764 \\
\hline Syzygium forte (F.Muell.) B.Hyland & Myrtaceae & 113 & 31 & 31.5 & 1.2134 & 1.3255 \\
\hline Syzygium malaccense (L.) Merr. and L.M.Perry & Myrtaceae & 91 & 33 & 31.8 & 2.2233 & 2.4616 \\
\hline
\end{tabular}


Table A2. Cont.

\begin{tabular}{ccccccc}
\hline Species & Family & Circ (cm) & Air T & Stem T & Sample 1 & Sample 2 \\
\hline Tamarindus indica L. & Fabaceae & 119 & 33 & 32.2 & 6.6644 & 7.5740 \\
Tecoma stans Griseb. & Bignoniaceae & 61 & 33 & 31.9 & 2.1645 & 2.3984 \\
Tectona grandis L.f. & Lamiaceae & 65 & 33 & 32.2 & 0.6566 & 0.6943 \\
Terminalia catappa L. & Combretaceae & 53 & 30 & 30.5 & 2.4844 & 2.7771 \\
Terminalia ivorensis A.Chev. & Combretaceae & 69 & 33 & 31.9 & 3.5467 & 3.9865 \\
Triplaris americana L. & Polygonaceae & 97 & 32 & 31.1 & 2.5546 & 2.8403 \\
Xanthostemon chrysanthus (F.Muell.) Benth. & Xanthorrhoeaceae & 96 & 33 & 32.2 & 3.6134 & 4.0395 \\
\hline
\end{tabular}

Table A3. List of non-palm arborescent monocot species included the in carbon dioxide efflux study. Circ $=$ circumference, Air $\mathrm{T}=$ air temperature, and Stem $\mathrm{T}=$ surface temperature of stems.

\begin{tabular}{|c|c|c|c|c|c|c|}
\hline Species & Family & Circ (cm) & Air T & Stem $\mathbf{T}$ & Sample 1 & Sample 2 \\
\hline Beaucarnea recurvata Lem. & Asparagaceae & 84 & 31 & 30.2 & 3.1848 & 2.8466 \\
\hline Dasylirion wheeleri S.Watson ex Rothr. & Asparagaceae & 99 & 33 & 32.6 & 4.4489 & 4.0032 \\
\hline Dracaena cochinchinensis (Lour.) S.C.Chen & Asparagaceae & 117 & 32 & 31.5 & 2.9441 & 2.6509 \\
\hline Dracaena dereminis Engl. & Asparagaceae & 58 & 33 & 32.5 & 1.2234 & 1.0730 \\
\hline Dracaena floribunda Baker & Asparagaceae & 101 & 33 & 32.2 & 2.0498 & 1.8304 \\
\hline Dracaena fragrans (L.) Ker Gaw. & Asparagaceae & 66 & 33 & 32.1 & 3.4899 & 3.0927 \\
\hline Ensete ventricosum (Welw.) Cheesman & Musaceae & 175 & 33 & 31.8 & 4.7003 & 4.2099 \\
\hline Musa $x$ paradisiaca $\mathrm{L}$. & Musaceae & 75 & 31 & 30.5 & 2.4468 & 2.6798 \\
\hline Pandanus dubius Spreng. & Pandanaceae & 73 & 32 & 31.7 & 3.5564 & 3.9888 \\
\hline Pandanus rabaiensis Rendle. & Pandanaceae & 64 & 33 & 32.1 & 3.9166 & 3.4714 \\
\hline Pandanus tectorius Parkinson ex Du Roi & Pandanaceae & 71 & 31 & 31.2 & 3.0865 & 3.4470 \\
\hline Pandanus utilis Bory & Pandanaceae & 58 & 32 & 31.8 & 0.7658 & 0.8205 \\
\hline Pandanus vandermeeschii Balf.f. & Pandanaceae & 68 & 31 & 30.3 & 3.8465 & 4.2920 \\
\hline Pandanus veitchii Mast. & Pandanaceae & 72 & 33 & 32.2 & 2.1779 & 2.3984 \\
\hline Ravenala madagascariensis Sonn. & Strelitziaceae & 85 & 31 & 30.6 & 1.0044 & 0.9468 \\
\hline Strelitzia alba (L.f.) Skeels & Strelitziaceae & 51 & 32 & 31.1 & 3.5668 & 4.0547 \\
\hline Xanthorrhoea glauca D.J.Bedford & Xanthorrhoeaceae & 78 & 31 & 31.5 & 1.4465 & 1.5779 \\
\hline
\end{tabular}

Table A4. List of the Arecaceae species included in the carbon dioxide efflux study. Circ = circumference, Air T = air temperature, and Stem $\mathrm{T}=$ surface temperature of stems.

\begin{tabular}{|c|c|c|c|c|c|}
\hline Species & Circ (cm) & Air T & Stem $\mathrm{T}$ & Sample 1 & Sample 2 \\
\hline Adonia merrillii (Becc.) Becc. & 66 & 33 & 31.9 & 1.4868 & 1.3255 \\
\hline Aiphanes minima (Gaertn.) Burret & 53 & 33 & 31.8 & 3.3622 & 3.6797 \\
\hline Allagoptera caudescens (Mart.) Kuntze & 52 & 32 & 30.8 & 1.6623 & 1.8051 \\
\hline Archontophoenix myolensis Dowe & 87 & 31 & 32.8 & 2.4465 & 2.1460 \\
\hline Archontophoenix purpurea Hodel and Dowe & 51 & 32 & 31.2 & 4.1657 & 3.6645 \\
\hline Areca catechu $\mathrm{L}$. & 59 & 32 & 31.8 & 1.8165 & 2.0134 \\
\hline Areca macrocarpa Becc. & 61 & 33 & 32.1 & 3.7799 & 4.3046 \\
\hline Areca parens Becc. & 59 & 33 & 33.9 & 3.2136 & 3.5649 \\
\hline Astrocaryum mexicanum Leibm. Ex Mart. & 51 & 32 & 31.1 & 2.5644 & 2.2722 \\
\hline $\begin{array}{c}\text { Beccariophoenix alfredii Rakotoarin., Ranariv. and } \\
\text { J.Dransf. }\end{array}$ & 182 & 33 & 32.9 & 1.1165 & 0.9864 \\
\hline Beccariophoenix madagascariensis Jum. and H.Perrier & 103 & 32 & 31.2 & 3.6645 & 4.0031 \\
\hline Bentinckia nicobarica (Kurz.) Becc. & 65 & 32 & 31.8 & 3.1619 & 3.4466 \\
\hline Borassodendron machadonis Becc. & 127 & 33 & 31.2 & 2.8645 & 3.1165 \\
\hline Brassiophoenix schumannii (Becc.) Essig & 52 & 33 & 32.1 & 1.7115 & 1.5274 \\
\hline Burretiokentia dumasii Pintaud and Hodel & 51 & 32 & 31.1 & 2.7654 & 3.0031 \\
\hline Burretiokentia grandiflora Pintaud and Hodel & 52 & 32 & 31.7 & 4.2958 & 4.7799 \\
\hline Burretiokentia vieillardii (Brongn. and Gris) Pic.Serm. & 51 & 33 & 31.9 & 0.8286 & 0.8898 \\
\hline
\end{tabular}


Table A4. Cont.

\begin{tabular}{|c|c|c|c|c|c|}
\hline Species & Circ $(\mathrm{cm})$ & Air T & Stem $\mathrm{T}$ & Sample 1 & Sample 2 \\
\hline Calyptrocalyx spicatus (Lam.) Blume & 52 & 34 & 32.9 & 1.2989 & 1.1361 \\
\hline Calyptronoma rivalis (O.F.Cook) L.H.Bailey & 66 & 33 & 31.7 & 2.6798 & 2.9765 \\
\hline Carpentaria acuminata (H.Wendl. and Drude) Becc. & 52 & 33 & 32.2 & 2.2722 & 1.7042 \\
\hline Carpoxylon macrospermum H.Wendl. and Drude & 67 & 32 & 30.9 & 0.9346 & 0.9979 \\
\hline Caryota ophiopellis Dowe & 81 & 32 & 31.1 & 3.9966 & 4.9643 \\
\hline Chambeyronia macrocarpa (Brongn.) Vieill. Ex Becc. & 51 & 33 & 32.1 & 1.8879 & 1.6410 \\
\hline Chelyocarpus chuco (Mart.) H.E.Moore & 51 & 32 & 31.1 & 3.6626 & 4.0033 \\
\hline Chelyocarpus ulei Dammer & 51 & 33 & 31.8 & 4.7474 & 5.2646 \\
\hline Clinostigma ponapense (Becc.) H.E.Moore and Fosberg & 62 & 33 & 32.1 & 3.6264 & 3.9986 \\
\hline Clinostigma samoense H.Wendl. & 68 & 33 & 31.8 & 4.8946 & 5.3970 \\
\hline Cocos nucifera L. & 105 & 32 & 31.4 & 3.7764 & 4.1133 \\
\hline Colpothrinax wrightii Griseb. and H.Wendl. ex Voss & 101 & 33 & 32.2 & 1.8445 & 1.6474 \\
\hline Copernicia baileyana León & 162 & 32 & 31.5 & 0.9631 & 0.9987 \\
\hline Copernicia hospita Mart. & 61 & 31 & 30.3 & 3.0844 & 3.4125 \\
\hline Copernicia prunifera (Mill.) H.E.Moore & 64 & 33 & 32.1 & 4.9497 & 5.4895 \\
\hline Copernicia sp. Mart. ex Endl. & 68 & 32 & 31.6 & 1.3346 & 1.4653 \\
\hline Corypha utan Lam. & 105 & 33 & 32.1 & 3.0888 & 3.4102 \\
\hline Cryosophila warscewiczii (H.Wendl.) Bartlett & 61 & 33 & 31.9 & 4.0054 & 3.6103 \\
\hline Cryosophila williamsii P.H.Allen & 52 & 32 & 31.1 & 5.4986 & 5.0896 \\
\hline $\begin{array}{c}\text { Cyphophoenix elegans (Brongn. and Gris) H.Wendl. ex } \\
\text { Salomon }\end{array}$ & 51 & 33 & 32.1 & 2.7298 & 2.3755 \\
\hline Cyphophoenix nucele H.E.Moore & 52 & 32 & 31.6 & 3.1959 & 3.0296 \\
\hline Cyrtostachys elegans Burret & 58 & 32 & 31.1 & 4.1121 & 3.6570 \\
\hline Cyrtostachys loriae Becc. & 52 & 33 & 32.1 & 3.1645 & 2.8165 \\
\hline Dictyosperma album (Bory) Scheff. & 58 & 32 & 31.3 & 1.3896 & 1.2497 \\
\hline Dypsis arenarum (Jum.) Beentje and J.Dransf. & 63 & 34 & 33.1 & 5.1132 & 4.5547 \\
\hline Dypsis cabadae (H.E.Moore) Beentje and J.Dransf. & 52 & 33 & 32.7 & 3.3132 & 2.9765 \\
\hline Dypsis carlsmithii J.Dransf. and Marcus & 77 & 33 & 32.9 & 4.6002 & 4.1165 \\
\hline Dypsis decaryi (Jum.) Beentje and J.Dransf. & 81 & 32 & 31.2 & 3.4497 & 3.1133 \\
\hline Dypsis hovomantsina Beentje & 62 & 33 & 33.7 & 1.3886 & 3.1349 \\
\hline Dypsis ifanadianae Beentje & 58 & 33 & 33.1 & 4.1546 & 3.8845 \\
\hline Dypsis lastelliana (Baill.) Beentje and J.Dransf. & 77 & 33 & 33.2 & 6.1132 & 5.6068 \\
\hline Dypsis madagascariensis (Becc.) Beentje and J.Dransf. & 55 & 34 & 33.5 & 5.7746 & 5.1770 \\
\hline $\begin{array}{c}\text { Dypsis mananjarensis (Jum. and H.Perrier) Beentje and } \\
\text { J.Dransf. }\end{array}$ & 65 & 34 & 31.9 & 3.8465 & 3.4545 \\
\hline Dypsis montana (Jum.) Beentje and J.Dransf. & 65 & 33 & 32.2 & 4.0064 & 3.6699 \\
\hline Dypsis pembana (H.E.Moore) Beentje and J.Dransf. & 68 & 29 & 28.1 & 4.5959 & 4.2236 \\
\hline Dypsis plumosa Hodel, J.Marcus and J.Dransf. & 62 & 32 & 31.5 & 1.9976 & 2.8965 \\
\hline Dypsis robusta Hodel, Marcus and J.Dransf. & 69 & 34 & 33.9 & 1.8465 & 1.6410 \\
\hline Dypsis saintelucei Beentje & 55 & 34 & 33.1 & 1.9292 & 1.7042 \\
\hline Elaeis guineensis Jacq. & 118 & 33 & 32.2 & 1.1645 & 1.0235 \\
\hline Euterpe precatoria Mart. & 51 & 32 & 31.4 & 2.4265 & 2.1775 \\
\hline Heterospathe elata Scheff. & 64 & 33 & 32.1 & 2.3164 & 2.1050 \\
\hline Heterospathe intermedia (Becc.) Fernando & 51 & 32 & 31.2 & 4.3465 & 3.9133 \\
\hline Heterospathe sibuyanensis Becc. & 74 & 34 & 31.9 & 2.0065 & 1.8304 \\
\hline Hydriastele moluccana (Becc.) W.J.Baker and Loo & 84 & 34 & 33.1 & 3.8844 & 3.4466 \\
\hline Hyophorbe lagenicaulis (L.H.Bailey) H.E.Moore & 151 & 31 & 30.4 & 2.8897 & 2.5878 \\
\hline Itaya amicorum H.E.Moore & 55 & 32 & 30.9 & 2.4246 & 2.1460 \\
\hline Kentiopsis piersoniorum Pintaud and Hodel & 55 & 33 & 32.1 & 1.4465 & 1.3255 \\
\hline Kentiopsis pyriformis Pintaud and Hodel & 65 & 32 & 31.2 & 3.6611 & 3.2821 \\
\hline Laccospadix australasicus H.Wendl. and Drude & 51 & 33 & 32.7 & 1.7996 & 1.5969 \\
\hline Licuala bayana Saw & 51 & 32 & 30.8 & 2.1132 & 1.8935 \\
\hline Licuala peltata Roxb. & 58 & 32 & 31.3 & 1.0054 & 0.8836 \\
\hline Licuala sallehana Saw & 69 & 30 & 29.1 & 1.6632 & 1.5148 \\
\hline Livistona lanuginosa Rodd & 97 & 33 & 32.1 & 2.8277 & 2.5247 \\
\hline
\end{tabular}


Table A4. Cont.

\begin{tabular}{|c|c|c|c|c|c|}
\hline Species & Circ (cm) & Air T & Stem $\mathrm{T}$ & Sample 1 & Sample 2 \\
\hline Livistona mariae F.Muell. & 88 & 33 & 32.3 & 1.1312 & 0.9957 \\
\hline Livistona muelleri F.M.Bailey & 74 & 32 & 31.5 & 1.8486 & 1.6410 \\
\hline Livistona victoriae Rodd & 79 & 31 & 30.9 & 1.6645 & 1.5148 \\
\hline Lodoicea maldivica (J.F.Gmel.) Pers. & 98 & 34 & 34.6 & 1.1132 & 0.9720 \\
\hline Medemia argun (Mart.) Wurttenb. ex H.Wendl. & 117 & 32 & 30.3 & 2.7918 & 2.5878 \\
\hline Neonicholsonia watsonii Drammer & 52 & 32 & 30.8 & 1.7765 & 1.5148 \\
\hline Neoveitchia brunnea Dowe & 55 & 33 & 31.8 & 2.0021 & 1.8051 \\
\hline Neoveitchia storckii (H.Wendl.) Becc. & 73 & 32 & 31.2 & 1.2345 & 1.1109 \\
\hline $\begin{array}{c}\text { Nephrosperma van-houtteanum (H.Wendl. ex Van Houtte) } \\
\text { Balf.f. }\end{array}$ & 52 & 33 & 32.3 & 2.7711 & 2.4657 \\
\hline Oenocarpus mapora H.Karst & 51 & 32 & 31.1 & 5.5056 & 4.9231 \\
\hline Orania moluccana Becc. & 68 & 29 & 28.6 & 1.6465 & 1.4391 \\
\hline Pelagodoxa henryana Becc. & 67 & 32 & 31.3 & 1.4215 & 1.6410 \\
\hline Phoenix sylvestris (L.) Roxb. & 109 & 31 & 32.9 & 3.7164 & 4.1132 \\
\hline Pinanga batanensis Becc. & 68 & 34 & 32.9 & 3.6134 & 4.0066 \\
\hline Pinanga insignis Becc. & 48 & 33 & 31.9 & 2.0311 & 1.8304 \\
\hline Pinanga javana Blume & 51 & 34 & 32.8 & 2.1798 & 1.9566 \\
\hline Pinanga urosperma Becc. & 61 & 33 & 31.9 & 0.9554 & 0.8205 \\
\hline Ponapea hosinoi Kaneh. & 62 & 33 & 31.8 & 5.4165 & 6.0023 \\
\hline Prestoea acuminata (Willd.) H.E.Moore & 52 & 32 & 30.8 & 4.0154 & 4.4651 \\
\hline Pritchardia thurstonii F.Muell. and Drude & 66 & 29 & 28.6 & 0.7879 & 0.6943 \\
\hline Ptychosperma elegans (R.Br.) Blume & 53 & 32 & 31.2 & 6.3897 & 7.1322 \\
\hline Ravenea madagascariensis Becc. & 52 & 34 & 33.2 & 0.9498 & 1.1021 \\
\hline Rhopaloblaste augusta (Kurz.) H.E.Mllre & 58 & 33 & 32.2 & 1.6355 & 1.4517 \\
\hline Rhopaloblaste ceramica (Miq.) Burret & 72 & 32 & 31.1 & 1.6899 & 1.5148 \\
\hline Sabal mauritiiformis (H.Karst.) Griseb. and H.Wendl. & 71 & 32 & 31.4 & 3.1134 & 3.2265 \\
\hline Sabal palmetto (Walter) Lodd. ex Schult. and Schult.f. & 95 & 31 & 31.3 & 1.3444 & 1.2231 \\
\hline Saribus rotundifolius (Lam.) Blume & 105 & 30 & 30.1 & 1.1314 & 1.0099 \\
\hline Satakentia liukiuensis (Hatus.) H.E.Moore & 68 & 32 & 31.5 & 7.5109 & 6.3365 \\
\hline Schippia concolor Burret & 51 & 33 & 32.4 & 0.7544 & 0.7547 \\
\hline Syagrus botryophora (Mart.) Mart. & 76 & 32 & 32.6 & 1.7655 & 1.5779 \\
\hline Syagrus romanzoffiana (Cham.) Glassman & 63 & 33 & 32.1 & 1.2615 & 1.1165 \\
\hline Syagrus sancona (Kunth.) H.Karst. & 78 & 32 & 31.8 & 1.6311 & 1.4517 \\
\hline Syagrus schizophylla (Mart.) Glassman & 56 & 32 & 31.1 & 1.5644 & 1.3886 \\
\hline Veitchia joannis H.Wendl. & 59 & 33 & 32.2 & 1.3433 & 1.1992 \\
\hline Washingtonia robusta $\mathrm{H}$.Wendl. & 154 & 32 & 31.3 & 4.6899 & 4.2288 \\
\hline Wodyetia bifurcata A.K.Irvine & 99 & 30 & 31.6 & 4.4454 & 3.9764 \\
\hline
\end{tabular}

\section{References}

1. Yang, J.; He, Y.; Aubrey, D.P.; Zhuang, Q.; Teskey, R.O. Global patterns and predictors of stem $\mathrm{CO}_{2}$ efflux in forest ecosystems. Glob. Chang. Biol. 2016, 22, 1433-1444. [CrossRef] [PubMed]

2. Vargas, R.; Barba, J. Greenhouse gas fluxes from tree stems. Trends Plant Sci. 2019, 24, 296-299. [CrossRef]

3. Cavaleri, M.A.; Oberbauer, S.F.; Ryan, M.G. Wood $\mathrm{CO}_{2}$ efflux in a primary tropical rain forest. Global Chang. Biol. 2006, 12, 2442-2458. [CrossRef]

4. Marler, T.E. Stem $\mathrm{CO}_{2}$ efflux of Cycas micronesica is reduced by chronic non-native insect herbivory. Plant Signal Behav. 2020, 15, 1716160. [CrossRef]

5. Marler, T.E.; Krishnapillai, M.V. Vertical strata and stem carbon dioxide efflux in Cycas trees. Plants 2020, 9, 230. [CrossRef]

6. Marler, T.E.; Lindström, A.J. Diel patterns of stem $\mathrm{CO}_{2}$ efflux vary among cycads, arborescent monocots, and woody eudicots and gymnosperms. Plant Signal Behav. 2020, 15, 1732661. [CrossRef]

7. Bloemen, J.; McGuire, M.A.; Aubrey, D.P.; Teskey, R.O.; Steppe, K. Transport of root-respired $\mathrm{CO}_{2}$ via the transpiration stream affects aboveground carbon assimilation and $\mathrm{CO}_{2}$ efflux in trees. New Phytol. 2013, 197, 555-565. [CrossRef] [PubMed]

8. Kunert, N. A case study on the vertical and diurnal variation of stem $\mathrm{CO}_{2}$ effluxes in an Amazonian forest tree. Trees 2018, 32, 913-917. [CrossRef]

9. Bowman, W.P.; Barbour, M.M.; Turnbull, M.H.; Tissue, D.T.; Whitehead, D.; Griffin, K.L. Sap flow rates and sapwood density are critical factors in within- and between-tree variation in $\mathrm{CO}_{2}$ efflux from stems of mature Dacrydium cupressinum trees. New Phytol. 2005, 167, 815-828. [CrossRef] 
10. McGuire, M.A.; Cerasoli, S.; Teskey, R.O. $\mathrm{CO}_{2}$ fluxes and respiration of branch segments of sycamore (Platanus occidentalis L.) examined at different sap velocities, branch diameters, and temperatures. J. Exp. Bot. 2007, 58, 2159-2168. [CrossRef] [PubMed]

11. Tarvainen, L.; Wallin, G.; Lim, H.; Linder, S.; Oren, R.; Ottosson Löfvenius, M.; Räntfors, M.; Tor-ngern, P.; Marshall, J. Photosynthetic refixation varies along the stem and reduces $\mathrm{CO}_{2}$ efflux in mature boreal Pinus sylvestris trees. Tree Physiol. 2018, 38, 558-569. [CrossRef]

12. Hilman, B.; Muhr, J.; Trumbore, S.E.; Kunert, N.; Carbone, M.S.; Yuval, P.; Wright, S.J.; Moreno, G.; Pérez-Priego, O.; Migliavacca, M.; et al. Comparison of $\mathrm{CO}_{2}$ and $\mathrm{O}_{2}$ fluxes demonstrate retention of respired $\mathrm{CO}_{2}$ in tree stems from a range of tree species. Biogeosciences 2019, 16, 177-191. [CrossRef]

13. Rowland, L.; da Costa, A.C.; Oliveira, A.A.; Oliveira, R.S.; Bittencourt, P.L.; Costa, P.B.; Giles, A.L.; Sosa, A.I.; Coughlin, I.; Godlee, J.L.; et al. Drought stress and tree size determine stem $\mathrm{CO}_{2}$ efflux in a tropical forest. New Phytol. 2018, 218, 1393-1405. [CrossRef]

14. Salomon, R.L.; De Roo, L.; Bodé, S.; Boeckx, P.; Steppe, K. Efflux and assimilation of xylem-transported $\mathrm{CO}_{2}$ in stems and leaves of tree species with different wood anatomy. Plant Cell Environ. 2021, 44, 3494-3508. [CrossRef] [PubMed]

15. Stutz, S.S.; Anderson, J. Inside out: Measuring the effect of wood anatomy on the efflux and assimilation of xylem-transported $\mathrm{CO}_{2}$. Plant Cell Environ. 2021, 44, 3490-3493. [CrossRef] [PubMed]

16. Stevenson, D.W. Radial growth in Beaucarnea recurvata. Am. J. Bot. 1980, 67, 476-489. [CrossRef]

17. Stevenson, D.W. Radial growth in the Cycadales. Am. J. Bot. 1980, 67, 465-475. [CrossRef]

18. Stevenson, D.W.; Fisher, J.B. The developmental relationship between primary and secondary thickening growth in Cordyline (Agavaceae). Bot. Gaz. 1980, 141, 264-268. [CrossRef]

19. Tomlinson, P.B. The Structural Biology of Palms; Clarendon Press: Oxford, UK, 1990.

20. Rudall, P. Lateral meristems and stem thickening growth in monocotyledons. Bot. Rev. 1991, 57, 150-163. [CrossRef]

21. Rudall, P. New records of secondary thickening in monocotyledons. IAWA J. 1995, 16, 261-268. [CrossRef]

22. Tomlinson, P.B.; Huggett, B.A. Cell longevity and sustained primary growth in palm stems. Am. J. Bot. 2012, 99, 1891-1902. [CrossRef] [PubMed]

23. Steppe, K.; Saveyn, A.; McGuire, M.A.; Lemeur, R.; Teskey, R.O. Resistance to radial $\mathrm{CO}_{2}$ diffusion contributes to between-tree variation in $\mathrm{CO}_{2}$ efflux of Populus deltoides stems. Funct. Plant Biol. 2007, 34, 785-792. [CrossRef] [PubMed]

24. Campioli, M.; Malhi, Y.; Vicca, S.; Luyssaert, S.; Papale, D.; Peñuelas, J.; Reichstein, M.; Migliavacca, M.; Arain, M.A.; Janssens, I.A. Evaluating the convergence between eddy-covariance and biometric methods for assessing carbon budgets of forests. Nat. Commun. 2016, 7, 13717. [CrossRef]

25. Fragniere, Y.; Bétrisey, S.; Cardinaux, L.; Stoffel, M.; Kozlowski, G. Fighting their last stand? A global analysis of the distribution and conservation status of gymnosperms. J. Biogeogr. 2015, 42, 809-820. [CrossRef]

26. Wikelski, M.; Cooke, S.J. Conservation physiology. Trends Ecol. Evol. 2006, 21, 38-46. [CrossRef]

27. Cooke, S.J.; O'Connor, C.M. Making conservation physiology relevant to policy makers and conservation practitioners. Conserv. Lett. 2010, 3, 159-166. [CrossRef]

28. Mahoney, J.L.; Klug, P.E.; Reed, W.L. An assessment of the US endangered species act recovery plans: Using physiology to support conservation. Conserv. Physiol. 2018, 6, coy036. [CrossRef]

29. Norstog, K.J.; Nicholls, T.J. The Biology of the Cycads; Cornell University Press: Ithaca, NY, USA, 1997. 